\title{
A consensus model for group decision making under interval type-2 fuzzy environment*
}

\author{
Xiao-xiong ZHANG ${ }^{\dagger}$, Bing-feng GE, Yue-jin TAN \\ (College of Information System and Management, National University of Defense Technology, Changsha 410073, China) \\ †E-mail: zxxandxx@163.com \\ Received June 24, 2015; Revision accepted Sept. 26, 2015; Crosschecked Dec. 30, 2015
}

\begin{abstract}
We propose a new consensus model for group decision making (GDM) problems, using an interval type-2 fuzzy environment. In our model, experts are asked to express their preferences using linguistic terms characterized by interval type- 2 fuzzy sets (IT2 FSs), because these can provide decision makers with greater freedom to express the vagueness in real-life situations. Consensus and proximity measures based on the arithmetic operations of IT2 FSs are used simultaneously to guide the decision-making process. The majority of previous studies have taken into account only the importance of the experts in the aggregation process, which may give unreasonable results. Thus, we propose a new feedback mechanism that generates different advice strategies for experts according to their levels of importance. In general, experts with a lower level of importance require a larger number of suggestions to change their initial preferences. Finally, we investigate a numerical example and execute comparable models and ours, to demonstrate the performance of our proposed model. The results indicate that the proposed model provides greater insight into the GDM process.
\end{abstract}

Key words: Group decision making (GDM), Interval type-2 fuzzy sets (IT2 FSs), Feedback mechanism http://dx.doi.org/10.1631/FITEE.1500198

CLC number: O159; O22

\section{Introduction}

Group decision making (GDM) problems commonly occur for situations in the real world. A GDM problem is defined as the problem of selecting the best solution from a given set of alternatives $X=\left\{x_{1}, x_{2}, \ldots, x_{n}\right\}$, according to a group of experts $E=\left\{e_{1}, e_{2}, \ldots, e_{m}\right\}$, based on their unique preferences $\left\{P_{1}, P_{2}, \ldots, P_{m}\right\}$. In general, experts are associated with various fields, and they may hold quite different or even contradictory opinions. Therefore, one important process involves reconciling all of these opinions to reach a consensus and then finding the best solution that is acceptable to all (Cabrerizo et al., 2013).

\footnotetext{
* Project supported by the National Natural Science Foundation of China (Nos. 71501182 and 71571185 )

(10) ORCID: Xiao-xiong ZHANG, http://orcid.org/0000-0002-35247543

(C) Zhejiang University and Springer-Verlag Berlin Heidelberg 2016
}

GDM problems consist of two main processes: consensus and selection (Cabrerizo et al., 2010). The consensus process is concerned with reaching a certain level of consensus among the groups. So far, various models have been proposed for GDM problems (Wang and $\mathrm{Li}, 2015$ ). In general, a feedback mechanism has been incorporated into these models, to provide advice to experts to improve the degree of consensus. However, there remain some aspects to be addressed. As an example, consider a heterogeneous situation. The majority of consensus models in the literature take into account only the importance of the experts in the aggregation process (Herrera-Viedma $e t$ al., 2014). However, experts belong to various fields, and their opinions may carry different weights throughout the consensus-reaching process. Therefore, it is reasonable to distinguish among experts when providing recommendations to reach the highest consensus level. It is logical to assume that experts with low levels of importance should receive more 
advice than those with high levels. With this motivation, we propose that a new feedback mechanism should be generated which considers the experts' different importance levels when addressing advice.

In most cases, it is difficult or impossible for experts to assign precise or numerical values when expressing their opinions (Cabrerizo et al., 2015a). Fuzzy set theory has been introduced to handle the imprecision and uncertainties in real-life situations (Sabahi and Akbarzadeh-T, 2014). However, most of the existing fuzzy methods focus only on type- 1 fuzzy sets (T1 FSs). Type-2 fuzzy sets (T2 FSs) can be treated as an extension of T1 FSs. In most cases, the computational burden is too heavy to apply T2 FSs to real-life problems (Mendel et al., 2006). Hence, interval type-2 fuzzy sets (IT2 FSs), a special case of general T2 FSs, must be considered. Previously, IT2 FSs have been applied widely in perceptual computing (Mendel et al., 2010), control systems (Wu and Mendel, 2011), and other fields (Feng et al., 2014). For example, Chen and Lee (2010) proposed a new approach for dealing with multi-attribute GDM problems, based on the arithmetic operations of IT2 FSs. Moharrer et al. (2015) proposed a novel twophase methodology based on IT2 FSs for modeling linguistic label perception. Although various applications of IT2 FSs exist in different fields, they have not been applied before to the consensus-reaching process for solving GDM problems.

This article proposes a new consensus model for GDM problems using an IT2 fuzzy environment, motivated by the extra room for flexibility that IT2 FSs can provide in real-life situations (Chen and Lee, 2010). Moreover, to improve on the methods of previous studies, a new feedback mechanism is generated, which is guided by the experts' levels of importance throughout the consensus-reaching process. Moreover, a practical example is investigated and comparable models are executed, to illustrate the practicality and feasibility of the proposed model.

\section{Interval type-2 fuzzy set}

The main aim of this section is to provide some basic definitions related to the IT2 FS framework and its corresponding numerical operations.

Definition 1 (Mendel et al., 2006; Wang et al., 2012)
A T2 FS $\tilde{\tilde{A}}$ in the universe of discourse $X$ can be represented by a type- 2 membership function $\mu_{\tilde{A}}$ as follows:

$$
\begin{aligned}
\tilde{\tilde{A}}= & \left\{\left((x, u), \mu_{\tilde{\tilde{A}}}(x, u)\right) \mid \forall x \in X,\right. \\
& \left.\forall u \in J_{X} \subseteq[0,1], 0 \leq \mu_{\tilde{\tilde{A}}}(x, u) \leq 1\right\},
\end{aligned}
$$

where $J_{X}$ denotes an interval in $[0,1]$. Moreover, the T2 FS $\tilde{\tilde{A}}$ can be represented in an alternative form as follows:

$$
\tilde{\tilde{A}}=\int_{x \in X} \int_{u \in J_{X}} \frac{\mu_{\tilde{A}}(x, u)}{(x, u)},
$$

where $\iint$ represents the union over all admissible $x$ and $u$.

Definition 2 (Mendel et al., 2006) Let $\tilde{\tilde{A}}$ be a T2 FS in the universe of discourse $X$ represented by a type- 2 membership function $\mu_{\tilde{\tilde{A}}}$. If all $\mu_{\tilde{\tilde{A}}}(x, u)=1$, then $\tilde{\tilde{A}}$ is called an IT2 FS and can be expressed as follows:

$$
\tilde{\tilde{A}}=\int_{x \in X} \int_{u \in J_{X}} \frac{1}{(x, u)},
$$

where $J_{X}$ has the same meaning as above.

Definition 3 (Mendel et al., 2006) The upper and lower membership functions of an IT2 FS are type-1 membership functions, respectively. Fig. 1 shows a trapezoidal IT2 FS.

$$
\begin{aligned}
\tilde{\tilde{A}}_{i}= & \left(\tilde{A}_{i}^{\mathrm{U}}, \tilde{A}_{i}^{\mathrm{L}}\right) \\
= & \left(\left(a_{i 1}^{\mathrm{U}}, a_{i 2}^{\mathrm{U}}, a_{i 3}^{\mathrm{U}}, a_{i 4}^{\mathrm{U}} ; H_{1}\left(\tilde{A}_{i}^{\mathrm{U}}\right), H_{2}\left(\tilde{A}_{i}^{\mathrm{U}}\right)\right),\right. \\
& \left.\left(a_{i 1}^{\mathrm{L}}, a_{i 2}^{\mathrm{L}}, a_{i 3}^{\mathrm{L}}, a_{i 4}^{\mathrm{L}} ; H_{1}\left(\tilde{A}_{i}^{\mathrm{L}}\right), H_{2}\left(\tilde{A}_{i}^{\mathrm{L}}\right)\right)\right),
\end{aligned}
$$

where $H_{j}\left(\tilde{A}_{i}^{\mathrm{U}}\right)$ is the membership value of the element $a_{i(j+1)}^{\mathrm{U}}$ in the upper trapezoidal membership function $\tilde{A}_{i}^{\mathrm{U}}, H_{j}\left(\tilde{A}_{i}^{\mathrm{L}}\right)$ is the membership value of the element $a_{i(j+1)}^{\mathrm{L}}$ in the lower trapezoidal membership function $\tilde{A}_{i}^{\mathrm{L}}$, and all of these membership values 
belong to $[0,1]$.

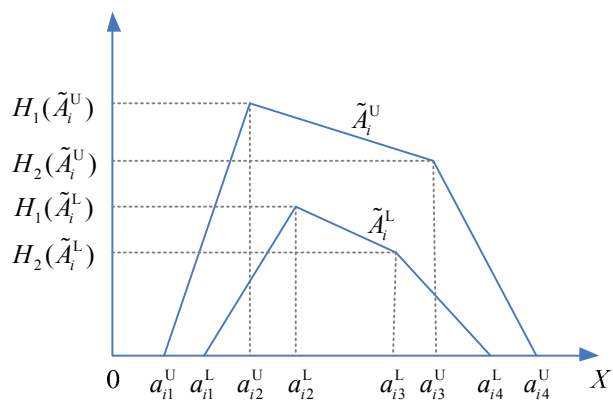

Fig. 1 Illustration of a trapezoidal IT2 FS

Definition 4 (Lee and Chen, 2008) Let

$$
\begin{aligned}
\tilde{\tilde{A}}_{1}=\left(\tilde{A}_{1}^{\mathrm{U}}, \tilde{A}_{1}^{\mathrm{L}}\right)= & \left(\left(a_{11}^{\mathrm{U}}, a_{12}^{\mathrm{U}}, a_{13}^{\mathrm{U}}, a_{14}^{\mathrm{U}} ; H_{1}\left(\tilde{A}_{1}^{\mathrm{U}}\right), H_{2}\left(\tilde{A}_{1}^{\mathrm{U}}\right)\right),\right. \\
& \left.\left(a_{11}^{\mathrm{L}}, a_{12}^{\mathrm{L}}, a_{13}^{\mathrm{L}}, a_{14}^{\mathrm{L}} ; H_{1}\left(\tilde{A}_{1}^{\mathrm{L}}\right), H_{2}\left(\tilde{A}_{1}^{\mathrm{L}}\right)\right)\right)
\end{aligned}
$$

and

$$
\begin{aligned}
\tilde{\tilde{A}}_{2}=\left(\tilde{A}_{2}^{\mathrm{U}}, \tilde{A}_{2}^{\mathrm{L}}\right)= & \left(\left(a_{21}^{\mathrm{U}}, a_{22}^{\mathrm{U}}, a_{23}^{\mathrm{U}}, a_{24}^{\mathrm{U}} ; H_{1}\left(\tilde{A}_{2}^{\mathrm{U}}\right), H_{2}\left(\tilde{A}_{2}^{\mathrm{U}}\right)\right),\right. \\
& \left.\left(a_{21}^{\mathrm{L}}, a_{22}^{\mathrm{L}}, a_{23}^{\mathrm{L}}, a_{24}^{\mathrm{L}} ; H_{1}\left(\tilde{A}_{2}^{\mathrm{L}}\right), H_{2}\left(\tilde{A}_{2}^{\mathrm{L}}\right)\right)\right)
\end{aligned}
$$

be two trapezoidal IT2 FSs. Then, some of the main operations between them are defined as follows:

$$
\begin{aligned}
& \tilde{\tilde{A}}_{1} \oplus \tilde{\tilde{A}}_{2}=\left(\tilde{A}_{1}^{\mathrm{U}}, \tilde{A}_{1}^{\mathrm{L}}\right) \oplus\left(\tilde{A}_{2}^{\mathrm{U}}, \tilde{A}_{2}^{\mathrm{L}}\right) \\
& =\left(\left(a_{11}^{\mathrm{U}}+a_{21}^{\mathrm{U}}, a_{12}^{\mathrm{U}}+a_{22}^{\mathrm{U}}, a_{13}^{\mathrm{U}}+a_{23}^{\mathrm{U}}, a_{14}^{\mathrm{U}}+a_{24}^{\mathrm{U}} ;\right.\right. \\
& \left.\min \left(H_{1}\left(\tilde{A}_{1}^{\mathrm{U}}\right), H_{1}\left(\tilde{A}_{2}^{\mathrm{U}}\right)\right), \min \left(H_{2}\left(\tilde{A}_{1}^{\mathrm{U}}\right), H_{2}\left(\tilde{A}_{2}^{\mathrm{U}}\right)\right)\right), \\
& \left(a_{11}^{\mathrm{L}}+a_{21}^{\mathrm{L}}, a_{12}^{\mathrm{L}}+a_{22}^{\mathrm{L}}, a_{13}^{\mathrm{L}}+a_{23}^{\mathrm{L}}, a_{14}^{\mathrm{L}}+a_{24}^{\mathrm{L}} ;\right. \\
& \left.\left.\min \left(H_{1}\left(\tilde{A}_{1}^{\mathrm{L}}\right), H_{1}\left(\tilde{A}_{2}^{\mathrm{L}}\right)\right), \min \left(H_{2}\left(\tilde{A}_{1}^{\mathrm{L}}\right), H_{2}\left(\tilde{A}_{2}^{\mathrm{L}}\right)\right)\right)\right), \\
& \tilde{\tilde{A}}_{1} \ominus \tilde{\tilde{A}}_{2}=\left(\tilde{A}_{1}^{\mathrm{U}}, \tilde{A}_{1}^{\mathrm{L}}\right) \ominus\left(\tilde{A}_{2}^{\mathrm{U}}, \tilde{A}_{2}^{\mathrm{L}}\right) \\
& =\left(\left(a_{11}^{\mathrm{U}}-a_{24}^{\mathrm{U}}, a_{12}^{\mathrm{U}}-a_{23}^{\mathrm{U}}, a_{13}^{\mathrm{U}}-a_{22}^{\mathrm{U}}, a_{14}^{\mathrm{U}}-a_{21}^{\mathrm{U}} ;\right.\right. \\
& \left.\min \left(H_{1}\left(\tilde{A}_{1}^{\mathrm{U}}\right), H_{1}\left(\tilde{A}_{2}^{\mathrm{U}}\right)\right), \min \left(H_{2}\left(\tilde{A}_{1}^{\mathrm{U}}\right), H_{2}\left(\tilde{A}_{2}^{\mathrm{U}}\right)\right)\right), \\
& \left(a_{11}^{\mathrm{L}}-a_{24}^{\mathrm{L}}, a_{12}^{\mathrm{L}}-a_{23}^{\mathrm{L}}, a_{13}^{\mathrm{L}}-a_{22}^{\mathrm{L}}, a_{14}^{\mathrm{L}}-a_{21}^{\mathrm{L}} ;\right. \\
& \left.\left.\min \left(H_{1}\left(\tilde{A}_{1}^{\mathrm{L}}\right), H_{1}\left(\tilde{A}_{2}^{\mathrm{L}}\right)\right), \min \left(H_{2}\left(\tilde{A}_{1}^{\mathrm{L}}\right), H_{2}\left(\tilde{A}_{2}^{\mathrm{L}}\right)\right)\right)\right),
\end{aligned}
$$

$$
\begin{aligned}
& \tilde{\tilde{A}}_{1} \otimes \tilde{\tilde{A}}_{2}=\left(\tilde{A}_{1}^{\mathrm{U}}, \tilde{A}_{1}^{\mathrm{L}}\right) \otimes\left(\tilde{A}_{2}^{\mathrm{U}}, \tilde{A}_{2}^{\mathrm{L}}\right) \\
& =\left(\left(a_{11}^{\mathrm{U}} \times a_{21}^{\mathrm{U}}, a_{12}^{\mathrm{U}} \times a_{22}^{\mathrm{U}}, a_{13}^{\mathrm{U}} \times a_{23}^{\mathrm{U}}, a_{14}^{\mathrm{U}} \times a_{24}^{\mathrm{U}} ;\right.\right. \\
& \left.\min \left(H_{1}\left(\tilde{A}_{1}^{\mathrm{U}}\right), H_{1}\left(\tilde{A}_{2}^{\mathrm{U}}\right)\right), \min \left(H_{2}\left(\tilde{A}_{1}^{\mathrm{U}}\right), H_{2}\left(\tilde{A}_{2}^{\mathrm{U}}\right)\right)\right), \\
& \left(a_{11}^{\mathrm{L}} \times a_{21}^{\mathrm{L}}, a_{12}^{\mathrm{L}} \times a_{22}^{\mathrm{L}}, a_{13}^{\mathrm{L}} \times a_{23}^{\mathrm{L}}, a_{14}^{\mathrm{L}} \times a_{24}^{\mathrm{L}} ;\right. \\
& \left.\left.\min \left(H_{1}\left(\tilde{A}_{1}^{\mathrm{L}}\right), H_{1}\left(\tilde{A}_{2}^{\mathrm{L}}\right)\right), \min \left(H_{2}\left(\tilde{A}_{1}^{\mathrm{L}}\right), H_{2}\left(\tilde{A}_{2}^{\mathrm{L}}\right)\right)\right)\right) .
\end{aligned}
$$

Definition 5 (Lee and Chen, 2008) Some arithmetic operations between the trapezoidal IT2 FSs are defined as follows:

$$
\begin{aligned}
k \tilde{\tilde{A}}_{1}= & \left(\left(k a_{11}^{\mathrm{U}}, k a_{12}^{\mathrm{U}}, k a_{13}^{\mathrm{U}}, k a_{14}^{\mathrm{U}} ; H_{1}\left(\tilde{A}_{1}^{\mathrm{U}}\right), H_{2}\left(\tilde{A}_{1}^{\mathrm{U}}\right)\right),\right. \\
& \left.\left(k a_{11}^{\mathrm{L}}, k a_{12}^{\mathrm{L}}, k a_{13}^{\mathrm{L}}, k a_{14}^{\mathrm{L}} ; H_{1}\left(\tilde{A}_{1}^{\mathrm{L}}\right), H_{2}\left(\tilde{A}_{1}^{\mathrm{L}}\right)\right)\right), \\
\frac{\tilde{A}_{1}}{k}= & \left(\left(\frac{a_{11}^{\mathrm{U}}}{k}, \frac{a_{12}^{\mathrm{U}}}{k}, \frac{a_{13}^{\mathrm{U}}}{k}, \frac{a_{14}^{\mathrm{U}}}{k} ; H_{1}\left(\tilde{A}_{1}^{\mathrm{U}}\right), H_{2}\left(\tilde{A}_{1}^{\mathrm{U}}\right)\right),\right. \\
& \left.\left(\frac{a_{11}^{\mathrm{L}}}{k}, \frac{a_{12}^{\mathrm{L}}}{k}, \frac{a_{13}^{\mathrm{L}}}{k}, \frac{a_{14}^{\mathrm{L}}}{k} ; H_{1}\left(\tilde{A}_{1}^{\mathrm{L}}\right), H_{2}\left(\tilde{A}_{1}^{\mathrm{L}}\right)\right)\right),
\end{aligned}
$$

Definition 6 (Lee and Chen, 2008) The ranking value $\operatorname{Rank}\left(\tilde{\tilde{A}}_{i}\right)$ of a trapezoidal IT2 FS $\tilde{\tilde{A}}_{i}$ is defined as follows:

$$
\begin{aligned}
& \operatorname{Rank}\left(\tilde{\tilde{A}}_{i}\right)= \\
& M_{1}\left(\tilde{A}_{i}^{\mathrm{U}}\right)+M_{1}\left(\tilde{A}_{i}^{\mathrm{L}}\right)+M_{2}\left(\tilde{A}_{i}^{\mathrm{U}}\right)+M_{2}\left(\tilde{A}_{i}^{\mathrm{L}}\right)+M_{3}\left(\tilde{A}_{i}^{\mathrm{U}}\right)+M_{3}\left(\tilde{A}_{i}^{\mathrm{L}}\right) \\
& -0.25\left(S_{1}\left(\tilde{A}_{i}^{\mathrm{U}}\right)+S_{1}\left(\tilde{A}_{i}^{\mathrm{L}}\right)+S_{2}\left(\tilde{A}_{i}^{\mathrm{U}}\right)+S_{2}\left(\tilde{A}_{i}^{\mathrm{L}}\right)\right. \\
& \left.\quad+S_{3}\left(\tilde{A}_{i}^{\mathrm{U}}\right)+S_{3}\left(\tilde{A}_{i}^{\mathrm{L}}\right)+S_{4}\left(\tilde{A}_{i}^{\mathrm{U}}\right)+S_{4}\left(\tilde{A}_{i}^{\mathrm{L}}\right)\right) \\
& +H_{1}\left(\tilde{A}_{i}^{\mathrm{U}}\right)+H_{1}\left(\tilde{A}_{i}^{\mathrm{L}}\right)+H_{2}\left(\tilde{A}_{i}^{\mathrm{U}}\right)+H_{2}\left(\tilde{A}_{i}^{\mathrm{L}}\right),
\end{aligned}
$$

where $M_{p}\left(\tilde{A}_{i}^{j}\right)(1 \leq p \leq 3)$ denotes the average value of the elements $a_{i p}^{j}$ and $a_{i(p+1)}^{j}, \quad S_{q}\left(\tilde{A}_{i}^{j}\right)(1 \leq q \leq 3)$ the standard deviation of the elements $a_{i p}^{j}$ and $a_{i(p+1)}^{j}$, and $S_{4}\left(\tilde{A}_{i}^{j}\right)$ the standard deviation of the elements $a_{i 1}^{j}, a_{i 2}^{j}, a_{i 3}^{j}, a_{i 4}^{j}$. That is,

$$
M_{p}\left(\tilde{A}_{i}^{j}\right)=\frac{a_{i p}^{j}+a_{i(p+1)}^{j}}{2}, 1 \leq p \leq 3, j \in\{\mathrm{U}, \mathrm{L}\}
$$




$$
\begin{gathered}
S_{q}\left(\tilde{A}_{i}^{j}\right)=\sqrt{\frac{1}{2} \sum_{k=q}^{q+1}\left(a_{i k}^{j}-M_{q}\left(\tilde{A}_{i}^{j}\right)\right)^{2}}, 1 \leq q \leq 3, j \in\{\mathrm{U}, \mathrm{L}\}, \\
S_{4}\left(\tilde{A}_{i}^{j}\right)=\sqrt{\frac{1}{4} \sum_{k=1}^{4}\left(a_{i k}^{j}-\frac{1}{4} \sum_{k=1}^{4} a_{i k}^{j}\right)^{2}}, j \in\{\mathrm{U}, \mathrm{L}\} .
\end{gathered}
$$

Note that a larger ranking value indicates a larger corresponding number.

Definition 7 (Zhang and Zhang, 2013) The Hamming distance between $\tilde{\tilde{A}}_{1}$ and $\tilde{\tilde{A}}_{2}$, denoted by $D\left(\tilde{\tilde{A}}_{1}, \tilde{\tilde{A}}_{2}\right)$, can be expressed as follows:

$$
D\left(\tilde{\tilde{A}}_{1}, \tilde{\tilde{A}}_{2}\right)=\frac{d_{1}+d_{2}}{8},
$$

where

$$
\begin{aligned}
d_{1}= & \left|a_{11}^{\mathrm{U}}-a_{21}^{\mathrm{U}}\right|+\left|a_{12}^{\mathrm{U}} \cdot H_{1}\left(\tilde{A}_{1}^{\mathrm{U}}\right)-a_{22}^{\mathrm{U}} \cdot H_{1}\left(\tilde{A}_{2}^{\mathrm{U}}\right)\right| \\
& +\left|a_{13}^{\mathrm{U}} \cdot H_{2}\left(\tilde{A}_{1}^{\mathrm{U}}\right)-a_{23}^{\mathrm{U}} \cdot H_{2}\left(\tilde{A}_{2}^{\mathrm{U}}\right)\right|+\left|a_{14}^{\mathrm{U}}-a_{24}^{\mathrm{U}}\right|
\end{aligned}
$$

and

$$
\begin{aligned}
d_{2}= & \left|a_{11}^{\mathrm{L}}-a_{21}^{\mathrm{L}}\right|+\left|a_{12}^{\mathrm{L}} \cdot H_{1}\left(\tilde{A}_{1}^{\mathrm{L}}\right)-a_{22}^{\mathrm{L}} \cdot H_{1}\left(\tilde{A}_{2}^{\mathrm{L}}\right)\right| \\
& +\left|a_{13}^{\mathrm{L}} \cdot H_{2}\left(\tilde{A}_{1}^{\mathrm{L}}\right)-a_{23}^{\mathrm{L}} \cdot H_{2}\left(\tilde{A}_{2}^{\mathrm{L}}\right)\right|+\left|a_{14}^{\mathrm{L}}-a_{24}^{\mathrm{L}}\right| .
\end{aligned}
$$

\section{Consensus model under IT2 fuzzy environment}

Various consensus models have been proposed for GDM problems in previous decades, the majority of which are based on consensus and proximity measures to guide the feedback mechanism. These two measures focus on guiding the consensusreaching process until a satisfactory consensus level is reached. However, when generating advice for the experts associated with a particular problem, such models ignore the importance levels of the opinions given by experts (Pérez et al., 2014). Meanwhile, note that consensus is meant as a full agreement at the beginning, which has been proven to be unreasonable and makes no sense. Experts may have trouble assigning crisp values to alternatives when dealing with GDM problems in most real-life situations. Hence, fuzzy set theory has been introduced to handle such uncertainties. However, in existing studies, most consensus models are based on T1 FSs, which would be incapable of handling some complex situations in comparison with T2 FSs.

To handle these issues, we propose a new consensus model, incorporating a new feedback mechanism in an IT2 fuzzy environment. The new feedback mechanism generates different advice strategies based on the experts' levels of importance, and IT2 FS can provide decision makers with more flexibility. To the best of our knowledge, this is the first time that such a framework has been presented. We believe that this can provide greater insight into the consensusreaching process.

The consensus model consists of several stages, as shown in Fig. 2. First, the problem is presented to the experts. Then, the experts express their unique assessments, based on their own understanding, using an IT2 fuzzy environment. During the consensusreaching process, consensus measures must be calculated to check whether the consensus level is satisfactory. If yes, then the selection process is applied to choose the appropriate solution from the given set of possibilities. Otherwise, the feedback mechanism is initiated, generating useful advice to the experts based on their levels of understanding of the problem. Several consensus rounds may be necessary to achieve a certain level of consensus. In the following subsections, different stages are presented in detail.

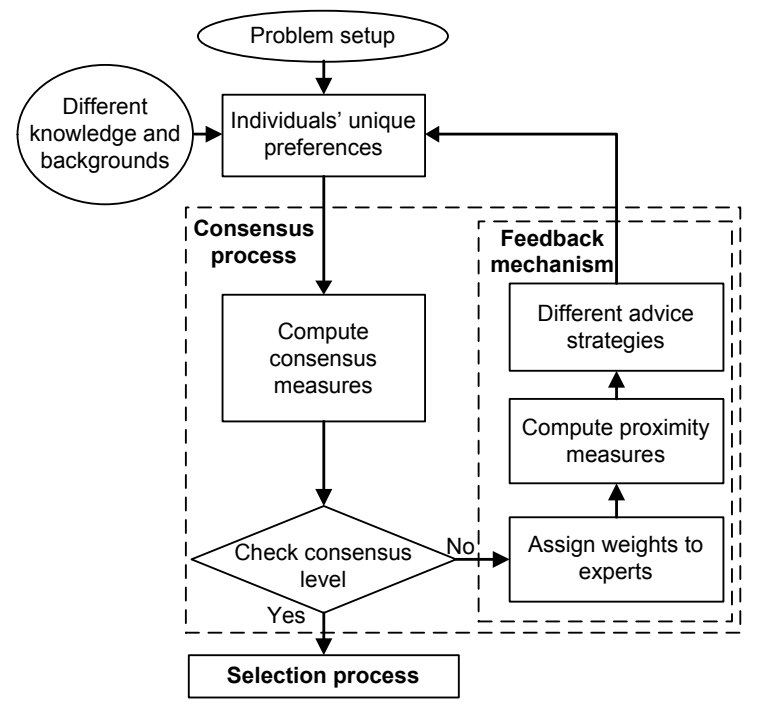

Fig. 2 New consensus model for group decision making (GDM) using an IT2 environment 


\subsection{Computing the consensus measures}

Suppose that each expert from a group $E=\left\{e_{1}\right.$, $\left.e_{2}, \ldots, e_{m}\right\}$ is required to express his/her fuzzy preferences $\left\{\tilde{\tilde{P}}_{1}, \tilde{\tilde{P}}_{2}, \ldots, \tilde{\tilde{P}}_{m}\right\}$ on a given set of alternatives $X=\left\{x_{1}, x_{2}, \ldots, x_{n}\right\}$, using the linguistic terms and their corresponding IT2 FSs shown in Table 1 (Chen and Lee, 2010). For an expert $e_{k}, \tilde{\tilde{p}}_{i j}^{k}$ denotes a preference for alternative $x_{i}$ over alternative $x_{j}$.

Table 1 Fuzzy linguistic assessment variables

\begin{tabular}{lc}
\hline \multicolumn{1}{c}{ Linguistic variable } & IT2 FS \\
\hline Very low (VL) & $((0,0,0,0.1 ; 1,1)$, \\
& $(0,0,0,0.05 ; 0.9,0.9))$ \\
Low $(\mathrm{L})$ & $((0,0.1,0.1,0.3 ; 1,1)$, \\
& $(0.05,0.1,0.1,0.2 ; 0.9,0.9))$ \\
Medium low (ML) & $((0.1,0.3,0.3,0.5 ; 1,1)$, \\
& $(0.2,0.3,0.3,0.4 ; 0.9,0.9))$ \\
Medium (M) & $((0.3,0.5,0.5,0.7 ; 1,1)$, \\
& $(0.4,0.5,0.5,0.6 ; 0.9,0.9))$ \\
Medium high (MH) & $((0.5,0.7,0.7,0.9 ; 1,1)$, \\
& $(0.6,0.7,0.7,0.8 ; 0.9,0.9))$ \\
High (H) & $((0.7,0.9,0.9,1 ; 1,1)$, \\
& $(0.8,0.9,0.9,0.95 ; 0.9,0.9))$ \\
Very high (VH) & $((0.9,1,1,1 ; 1,1)$, \\
& $(0.95,1,1,1 ; 0.9,0.9))$ \\
\hline
\end{tabular}

When the preferences have been provided, the consensus measures need to be calculated to ascertain whether the current consensus level in the decisionmaking process is satisfactory. In general, the consensus measures can be calculated at three different levels (Herrera-Viedma et al., 2007; Pérez et al., 2014; Zhang et al., 2015):

1. Initially, a similarity matrix $\mathbf{S M}^{k l}=\left(\mathrm{sm}_{i j}^{k l}\right)$, measuring the agreement between each pair of experts $\left(e^{k}, e^{l}\right)(k=1,2, \ldots, m-1 ; l=k+1, k+2, \ldots, m)$, is constructed as follows:

$$
\operatorname{sm}_{i j}^{k l}=1-D\left(\tilde{\tilde{p}}_{i j}^{k}, \tilde{\tilde{p}}_{i j}^{l}\right)
$$

where $D\left(\tilde{\tilde{p}}_{i j}^{k}, \tilde{\tilde{p}}_{i j}^{l}\right)$ is the distance between $\tilde{\tilde{p}}_{i j}^{k}$ and $\tilde{\tilde{p}}_{i j}^{l}$, calculated by using Eq. (11).

2. Subsequently, all of the constructed similarity matrices must be combined to obtain an aggregated consensus matrix, $\mathbf{C M}=\left(\mathrm{cm}_{i j}\right)$, using the following relationship (Pérez et al., 2014):

$$
\mathrm{cm}_{i j}=2 \sum_{k=1}^{m-1} \sum_{l=k+1}^{m} \frac{\mathrm{sm}_{i j}^{k l}}{m(m-1)} .
$$

It is held that $\mathrm{cm}_{i j} \in[0,1]$. The lower and upper bounds indicate the presence of no consensus and a total consensus for preference $p_{i j}$, respectively.

3 . Finally, the consensus measures at three different levels are defined as follows:

Consensus measure on preference:

$$
\mathrm{cd}_{i j}=\mathrm{cm}_{i j}
$$

Consensus measure on alternatives:

$$
\mathrm{CD}_{i}=\sum_{j=1, j \neq i}^{n} \frac{\mathrm{cd}_{i j}+\mathrm{cd}_{j i}}{2(n-1)} .
$$

Consensus measure on the relation:

$$
\mathrm{CD}=\sum_{i=1}^{n} \frac{\mathrm{CD}_{i}}{n} .
$$

After the consensus measure CD is obtained, it must be compared with a threshold $\mathrm{CR} \in[0,1]$, which is set beforehand as the minimum required consensus level. When $\mathrm{CD} \geq \mathrm{CR}$, the consensus-reaching process is acceptable, and the model moves onto the selection process. Otherwise, the feedback mechanism is activated, to aid the experts in changing their preferences and to narrow their differences to reach a higher consensus level. Note that a maximum number of repetitions should be decided, in case $\mathrm{CD}$ never converges to CR (Mata et al., 2009).

\subsection{Feedback mechanism}

As mentioned above, we propose a new feedback mechanism to guide the experts in changing their preferences based on their levels of importance. In general, experts with low levels of importance require more advice than those with high levels. Thus, in this study, we need to classify the experts into different levels in advance.

In this regard, the weights of different experts need to be determined first. However, in most situations, it is impossible to determine weights for the experts. Therefore, we assign the experts different 
weights directly using the preferences expressed by them. Following Chen and Yang (2011), the closer an expert's preference value is to the mean value, the larger the weight that should be assigned.

The mean preference value $\overline{\tilde{\tilde{p}}}_{i j}$ between two alternatives $x_{i}$ and $x_{j}$ is computed using Eq. (17):

$$
\overline{\tilde{\tilde{p}}}_{i j}=\frac{1}{k} \sum_{k=1}^{m} \tilde{\tilde{p}}_{i j}^{k}, i, j=1,2, \ldots, n .
$$

Then, we compute the similarity between the preference of each expert and the mean preference as in Eq. (18):

$$
S_{k}=1-\frac{1}{n(n-1)} \sum_{i=1}^{n} \sum_{j=1, j \neq i}^{n} D\left(\tilde{\tilde{p}}_{i j}^{k}, \overline{\tilde{\tilde{p}}}_{i j}\right) .
$$

The weight of expert $e_{k}$ is computed as follows:

$$
w_{k}=\frac{S_{k}}{\sum_{i=1}^{m} S_{i}} \text {. }
$$

Finally, we must classify the experts into three levels: low, medium, and high. The performance of this classification depends on the specific problem being dealt with.

After we obtain the weights and preferences of all experts, we combine all of the different comparison matrices into a single matrix for the group, $\tilde{\tilde{\boldsymbol{P}}}=\left(\tilde{\tilde{p}}_{i j}\right)$, using the following formula:

$$
\tilde{\tilde{p}}_{i j}=\sum_{k=1}^{m} w_{k} \tilde{\tilde{p}}_{i j}^{k} .
$$

Proximity measures are designed to measure the agreement between each individual's preference and the collective group preference. This can be used to guide the feedback mechanism. We proceed by computing this metric at three different levels, as shown below:

Proximity measure on preferences of each expert $\mathbf{P M}^{k}=\left(\mathrm{pm}_{i j}^{k}\right)$ :

$$
\operatorname{pm}_{i j}^{k}=1-D\left(\tilde{\tilde{p}}_{i j}^{k}, \tilde{\tilde{p}}_{i j}\right) .
$$

Proximity measure on alternatives:

$$
\mathrm{PM}_{i}^{k}=\frac{1}{2(n-1)} \sum_{j=1, j \neq i}^{n}\left(\mathrm{pm}_{i j}^{k}+\mathrm{pm}_{j i}^{k}\right) .
$$

Proximity measure on the relation:

$$
\mathrm{PM}^{k}=\frac{1}{n} \sum_{i=1}^{n} \mathrm{PM}_{i}^{k}
$$

Then, we propose three different strategies for providing advice to the experts with different importance levels (Zhang et al., 2015), after all of the related parameters have been calculated and obtained.

(a) For low-importance experts:

For experts at this level, we assume that they have little knowledge associated with the specific problem. Therefore, it is logical to assume that significant changes should be suggested by the feedback mechanism. To do this, we attempt to modify all of the preference values for which the consensus degree based on consensus measures is not high enough, as follows:

$$
R_{\text {Low }}^{k}=\left\{(i, j) \mid \mathrm{cd}_{i j}<\alpha_{1}\right\},
$$

where $\alpha_{1}=\frac{1}{n(n-1)} \sum_{i=1}^{n} \sum_{j=1, j \neq i}^{n} \mathrm{~cd}_{i j}$.

(b) For medium-importance experts

For experts at this level, fewer changes should be encouraged in comparison with the lowimportance experts. Therefore, we focus on disagreements at the level of alternatives (Pérez et al., 2014). To do this, a vector $\boldsymbol{\beta}=\left[\beta_{i}\right]$ is determined, where $\beta_{i}=\sum_{k=1}^{m} \mathrm{PM}_{i}^{k} / m(i=1,2, \ldots, n)$.

We then define the preference values that need to be changed, based on consensus measures in conjunction with the proximity measures, as follows:

$$
R_{\mathrm{Med}}^{k}=\left\{(i, j) \mid \mathrm{cd}_{i j}<\alpha_{1} \cap \mathrm{CD}_{i}<\mathrm{CD} \cap \mathrm{PM}_{i}^{k}<\beta_{i}\right\} .
$$

(c) For high-importance experts

In general, the opinions of experts with higher levels of importance are more valuable, and it is 
logical to assume that their preferences should be preserved as much as possible, so that they can exert an influence on others. Thus, in this situation, we focus on the preference values that hinder agreement with the collective preference at pairs of alternatives (Pérez et al., 2014), based on the proximity measures:

$$
\begin{aligned}
R_{\text {High }}^{k}= & \left\{(i, j) \mid \mathrm{cd}_{i j}<\alpha_{1} \cap \mathrm{CD}_{i}<\mathrm{CD}\right. \\
& \left.\cap \mathrm{PM}_{i}^{k}<\beta_{i} \cap \mathrm{pm}_{i j}^{k}<\alpha_{2}\right\},
\end{aligned}
$$

where

$$
\alpha_{2}=\frac{\sum_{i=1}^{n} \sum_{j=1, j \neq i}^{n} \mathrm{pm}_{i j}^{k}}{n(n-1)} .
$$

Once all of the preference values that need to be changed have been identified, different steps should then be followed in different cases. In this regard, an expert should increase the assessment when $\tilde{\tilde{p}}_{i j}^{k}<\tilde{\tilde{p}}_{i j}$ or decrease it when $\tilde{\tilde{p}}_{i j}^{k}>\tilde{\tilde{p}}_{i j}$.

\subsection{Selection process}

After a certain number of consensus rounds have been carried out and an acceptable agreement level is reached, i.e., $C D \geq C R$, the selection process begins.

When we obtain the collective preference matrix $\tilde{\tilde{\boldsymbol{P}}}$, the alternatives can be furnished with the ranking values. As previously mentioned, a larger ranking value often corresponds to a larger trapezoidal IT2 FS Hence, we define the dominance degree $\mathrm{px}_{i}$ of each alternative $x_{i}$ as in the following equation:

$$
\mathrm{px}_{i}=\sum_{j=1, j \neq i}^{n} \operatorname{Rank}\left(\tilde{\tilde{p}}_{i j}\right)
$$

Finally, a ranking order can be obtained for all the alternatives, and the alternative with the highest value for $\mathrm{px}_{i}$ is the best solution.

\section{Illustrative example}

\subsection{Proposed consensus model}

In this section, we use an example to show the performance of the proposed model. Suppose that four experts from different fields are invited to make judgments between four alternatives, using the fuzzy linguistic terms in Table 1. Following a period of consideration, the preferences were obtained as follows:

$$
\boldsymbol{E}^{1}=\left[\begin{array}{cccc}
- & \mathrm{VH} & \mathrm{VH} & \mathrm{VH} \\
\mathrm{VL} & - & \mathrm{MH} & \mathrm{H} \\
\mathrm{L} & \mathrm{ML} & - & \mathrm{M} \\
\mathrm{L} & \mathrm{L} & \mathrm{M} & -
\end{array}\right],
$$$$
\boldsymbol{E}^{2}=\left[\begin{array}{cccc}
- & \mathrm{VL} & \mathrm{ML} & \mathrm{ML} \\
\mathrm{VH} & - & \mathrm{H} & \mathrm{VH} \\
\mathrm{MH} & \mathrm{L} & - & \mathrm{M} \\
\mathrm{H} & \mathrm{VL} & \mathrm{M} & -
\end{array}\right] \text {, }
$$$$
\boldsymbol{E}^{3}=\left[\begin{array}{cccc}
- & \mathrm{M} & \mathrm{ML} & \mathrm{ML} \\
\mathrm{M} & - & \mathrm{L} & \mathrm{L} \\
\mathrm{H} & \mathrm{H} & - & \mathrm{M} \\
\mathrm{MH} & \mathrm{MH} & \mathrm{M} & -
\end{array}\right],
$$

$$
\boldsymbol{E}^{4}=\left[\begin{array}{cccc}
- & \mathrm{M} & \mathrm{ML} & \mathrm{ML} \\
\mathrm{M} & - & \mathrm{L} & \mathrm{L} \\
\mathrm{H} & \mathrm{H} & - & \mathrm{M} \\
\mathrm{MH} & \mathrm{MH} & \mathrm{M} & -
\end{array}\right] .
$$

The four linguistic matrices were then transformed into their corresponding IT2 FSs, as follows:

$$
\begin{gathered}
\tilde{\tilde{\boldsymbol{P}}}^{1}=\left[\begin{array}{cccc}
- & \tilde{\tilde{p}}_{12}^{1} & \tilde{\tilde{p}}_{13}^{1} & \tilde{\tilde{p}}_{14}^{1} \\
\tilde{\tilde{p}}_{21}^{1} & - & \tilde{\tilde{p}}_{23}^{1} & \tilde{\tilde{p}}_{24}^{1} \\
\tilde{\tilde{p}}_{31}^{1} & \tilde{\tilde{p}}_{32}^{1} & - & \tilde{\tilde{p}}_{34}^{1} \\
\tilde{\tilde{p}}_{41}^{1} & \tilde{\tilde{p}}_{42}^{1} & \tilde{\tilde{p}}_{43}^{1} & -
\end{array}\right], \tilde{\tilde{\boldsymbol{P}}}^{2}=\left[\begin{array}{cccc}
- & \tilde{\tilde{p}}_{12}^{2} & \tilde{\tilde{p}}_{13}^{2} & \tilde{\tilde{p}}_{14}^{2} \\
\tilde{\tilde{p}}_{21}^{2} & - & \tilde{\tilde{p}}_{23}^{2} & \tilde{\tilde{p}}_{24}^{2} \\
\tilde{\tilde{p}}_{31}^{2} & \tilde{\tilde{p}}_{32}^{2} & - & \tilde{\tilde{p}}_{34}^{2} \\
\tilde{\tilde{p}}_{41}^{2} & \tilde{\tilde{p}}_{42}^{2} & \tilde{\tilde{p}}_{43}^{2} & -
\end{array}\right], \\
\tilde{\tilde{\boldsymbol{P}}}^{3}=\left[\begin{array}{ccccc}
- & \tilde{\tilde{p}}_{12}^{3} & \tilde{\tilde{p}}_{13}^{3} & \tilde{\tilde{p}}_{14}^{3} \\
\tilde{\tilde{p}}_{31}^{3} & - & \tilde{\tilde{p}}_{23}^{3} & \tilde{\tilde{p}}_{24}^{3} \\
\tilde{\tilde{p}}_{31}^{3} & \tilde{\tilde{p}}_{32}^{3} & - & \tilde{\tilde{p}}_{34}^{3} \\
\tilde{\tilde{p}}_{41}^{3} & \tilde{\tilde{p}}_{42}^{3} & \tilde{\tilde{p}}_{43}^{3} & -
\end{array}\right], \tilde{\tilde{\boldsymbol{P}}}^{4}=\left[\begin{array}{cccc}
- & \tilde{\tilde{p}}_{12}^{4} & \tilde{\tilde{p}}_{13}^{4} & \tilde{\tilde{p}}_{14}^{4} \\
\tilde{\tilde{p}}_{21}^{4} & - & \tilde{\tilde{p}}_{23}^{4} & \tilde{\tilde{p}}_{24}^{4} \\
\tilde{\tilde{p}}_{31}^{4} & \tilde{\tilde{p}}_{32}^{4} & - & \tilde{\tilde{p}}_{34}^{4} \\
\tilde{\tilde{p}}_{41}^{4} & \tilde{\tilde{p}}_{42}^{4} & \tilde{\tilde{p}}_{43}^{4} & -
\end{array}\right] .
\end{gathered}
$$

Take $\tilde{\tilde{\boldsymbol{P}}}^{1}$, for instance, where

$$
\begin{aligned}
& \tilde{\tilde{p}}_{12}^{1}=((0.9,1,1,1 ; 1,1),(0.95,1,1,1 ; 0.9,0.9)), \\
& \tilde{\tilde{p}}_{13}^{1}=((0.9,1,1,1 ; 1,1),(0.95,1,1,1 ; 0.9,0.9)), \\
& \tilde{\tilde{p}}_{14}^{1}=((0.9,1,1,1 ; 1,1),(0.95,1,1,1 ; 0.9,0.9)),
\end{aligned}
$$


$\tilde{\tilde{p}}_{21}^{1}=((0,0,0,0.1 ; 1,1),(0,0,0,0.05 ; 0.9,0.9))$,

$\tilde{\tilde{p}}_{23}^{1}=((0.5,0.7,0.7,0.9 ; 1,1),(0.6,0.7,0.7,0.8 ; 0.9,0.9))$,

$\tilde{\tilde{p}}_{24}^{1}=((0.7,0.9,0.9,1 ; 1,1),(0.8,0.9,0.9,0.95 ; 0.9,0.9))$,

$\tilde{\tilde{p}}_{31}^{1}=((0,0.1,0.1,0.3 ; 1,1),(0.05,0.1,0.1,0.2 ; 0.9,0.9))$,

$\tilde{\tilde{p}}_{32}^{1}=((0.1,0.3,0.3,0.5 ; 1,1),(0.2,0.3,0.3,0.4 ; 0.9,0.9))$,

$\tilde{\tilde{p}}_{34}^{1}=((0.3,0.5,0.5,0.7 ; 1,1),(0.4,0.5,0.5,0.6 ; 0.9,0.9))$,

$\tilde{\tilde{p}}_{41}^{1}=((0,0.1,0.1,0.3 ; 1,1),(0.05,0.1,0.1,0.2 ; 0.9,0.9))$,

$\tilde{\tilde{p}}_{42}^{1}=((0,0.1,0.1,0.3 ; 1,1),(0.05,0.1,0.1,0.2 ; 0.9,0.9))$,

$\tilde{\tilde{p}}_{43}^{1}=((0.3,0.5,0.5,0.7 ; 1,1),(0.4,0.5,0.5,0.6 ; 0.9,0.9))$.

1. Computing the consensus measures

First, the six similarity matrices $\mathbf{S} \mathbf{M}^{12}, \mathbf{S M}^{13}$, $\mathbf{S M}{ }^{14}, \mathbf{S M}^{23}, \mathbf{S M}^{24}$, and $\mathbf{S M}^{34}$ were obtained by computing the agreement between each pair of experts using Eq. (12). Subsequently, a consensus matrix $\mathbf{C M}$ was obtained using Eq. (13). Then, the consensus measures at the three different levels were obtained using Eqs. (14)-(16).

Consensus measure on preference:

$$
\mathbf{C D}=\left[\begin{array}{cccc}
- & 0.53 & 0.67 & 0.67 \\
0.53 & - & 0.53 & 0.46 \\
0.60 & 0.53 & - & 1 \\
0.63 & 0.57 & 1 & -
\end{array}\right]
$$

Consensus measure on alternatives:

$$
\mathrm{CD}_{1}=0.61, \mathrm{CD}_{2}=0.53, \mathrm{CD}_{3}=0.72, \mathrm{CD}_{4}=0.72 \text {. }
$$

Consensus measure on the relation:

$$
\mathrm{CD}=\frac{1}{4} \sum_{i=1}^{4} \mathrm{CD}_{i}=0.645
$$

We set $\mathrm{CL}=0.7$, and see that $\mathrm{CD}<\mathrm{CL}$. This means that the consensus level was unacceptable. Thus, the feedback mechanism was activated.

2. Feedback mechanism

First, the weights of all of the experts were determined by using Eqs. (17)-(19).

The results were $w_{1}=0.22, w_{2}=0.24, w_{3}=0.27$, and $w_{4}=0.27$. In this problem, we set experts $e_{3}$ and $e_{4}$ as high-importance experts, $e_{2}$ as a mediumimportance expert, and $e_{1}$ as a low-importance expert.
After determining all of the experts' weights and their corresponding preferences, a global final comparison matrix was obtained by using Eq. (20), as follows:

$$
\tilde{\tilde{\boldsymbol{P}}}=\left[\begin{array}{cccc}
- & \tilde{\tilde{p}}_{12} & \tilde{\tilde{p}}_{13} & \tilde{\tilde{p}}_{14} \\
\tilde{\tilde{p}}_{21} & - & \tilde{\tilde{p}}_{23} & \tilde{\tilde{p}}_{24} \\
\tilde{\tilde{p}}_{31} & \tilde{\tilde{p}}_{32} & - & \tilde{\tilde{p}}_{34} \\
\tilde{\tilde{p}}_{41} & \tilde{\tilde{p}}_{42} & \tilde{\tilde{p}}_{43} & -
\end{array}\right],
$$

where

$$
\begin{aligned}
& \tilde{\tilde{p}}_{12}=((0.36,0.49,0.49,0.62 ; 1,1) \text {, } \\
& (0.43,0.49,0.49,0.56 ; 0.9,0.9)) \text {, } \\
& \tilde{\tilde{p}}_{13}=((0.28,0.45,0.45,0.61 ; 1,1) \text {, } \\
& (0.37,0.45,0.45,0.53 ; 0.9,0.9)) \text {, } \\
& \tilde{\tilde{p}}_{14}=((0.28,0.45,0.45,0.61 ; 1,1), \\
& (0.37,0.45,0.45,0.53 ; 0.9,0.9)) \text {, } \\
& \tilde{\tilde{p}}_{21}=((0.38,0.51,0.51,0.64 ; 1,1), \\
& (0.44,0.51,0.51,0.58 ; 0.9,0.9)) \text {, } \\
& \tilde{\tilde{p}}_{23}=((0.28,0.42,0.42,0.6 ; 1,1) \text {, } \\
& (0.35,0.42,0.42,0.51 ; 0.9,0.9)) \text {, } \\
& \tilde{\tilde{p}}_{24}=((0.37,0.49,0.49,0.62 ; 1,1) \text {, } \\
& (0.43,0.49,0.49,0.56 ; 0.9,0.9)) \text {, } \\
& \tilde{\tilde{p}}_{31}=((0.50,0.68,0.68,0.82 ; 1,1) \text {, } \\
& (0.59,0.68,0.68,0.75 ; 0.9,0.9)) \text {, } \\
& \tilde{\tilde{p}}_{32}=((0.4,0.58,0.58,0.72 ; 1,1) \text {, } \\
& (0.49,0.58,0.58,0.65 ; 0.9,0.9)) \text {, } \\
& \tilde{\tilde{p}}_{34}=((0.3,0.5,0.5,0.7 ; 1,1) \text {, } \\
& (0.4,0.5,0.5,0.6 ; 0.9,0.9)) \text {, } \\
& \tilde{\tilde{p}}_{41}=((0.44,0.62,0.62,0.79 ; 1,1) \text {, } \\
& (0.53,0.62,0.62,0.70 ; 0.9,0.9)) \text {, } \\
& \tilde{\tilde{p}}_{42}=((0.27,0.4,0.4,0.58 ; 1,1), \\
& (0.34,0.4,0.4,0.49 ; 0.9,0.9)) \text {, } \\
& \tilde{\tilde{p}}_{43}=((0.3,0.5,0.5,0.7 ; 1,1) \text {, } \\
& (0.4,0.5,0.5,0.6 ; 0.9,0.9)) \text {. }
\end{aligned}
$$

Proximity measures for each expert were then calculated using Eqs. (21)-(23):

Proximity measure on preference: 
$$
\begin{aligned}
\mathbf{P M}^{1} & =\left[\begin{array}{cccc}
- & 0.52 & 0.48 & 0.48 \\
0.52 & - & 0.74 & 0.62 \\
0.46 & 0.74 & - & 1.0 \\
0.52 & 0.72 & 1.0 & -
\end{array}\right], \\
\mathbf{P M}^{2} & =\left[\begin{array}{cccc}
- & 0.54 & 0.85 & 0.85 \\
0.54 & - & 0.56 & 0.53 \\
0.97 & 0.56 & - & 1.0 \\
0.74 & 0.62 & 1.0 & -
\end{array}\right], \\
\mathbf{P M}^{3} & =\left[\begin{array}{cccc}
- & 0.97 & 0.85 & 0.85 \\
0.97 & - & 0.70 & 0.64 \\
0.79 & 0.70 & - & 1.0 \\
0.92 & 0.72 & 1.0 & -
\end{array}\right], \\
\mathbf{P M}^{4} & =\left[\begin{array}{cccc}
- & 0.97 & 0.85 & 0.85 \\
0.97 & - & 0.70 & 0.64 \\
0.79 & 0.70 & - & 1.0 \\
0.92 & 0.72 & 1.0 & -
\end{array}\right] .
\end{aligned}
$$

Proximity measure on alternatives:

$$
\begin{aligned}
& \mathrm{PM}_{1}^{1}=0.50, \mathrm{PM}_{2}^{1}=0.64, \mathrm{PM}_{3}^{1}=0.74, \mathrm{PM}_{4}^{1}=0.72, \\
& \mathrm{PM}_{1}^{2}=0.75, \mathrm{PM}_{2}^{2}=0.56, \mathrm{PM}_{3}^{2}=0.82, \mathrm{PM}_{4}^{2}=0.79, \\
& \mathrm{PM}_{1}^{3}=0.89, \mathrm{PM}_{2}^{3}=0.78, \mathrm{PM}_{3}^{3}=0.84, \mathrm{PM}_{4}^{3}=0.85, \\
& \mathrm{PM}_{1}^{4}=0.89, \mathrm{PM}_{2}^{4}=0.78, \mathrm{PM}_{3}^{4}=0.84, \mathrm{PM}_{4}^{4}=0.85 .
\end{aligned}
$$

Proximity measure on relations:

$$
\mathrm{PM}^{1}=0.65, \mathrm{PM}^{2}=0.73, \mathrm{PM}^{3}=0.84, \mathrm{PM}^{4}=0.84 \text {. }
$$

Based on the rules and methods detailed above, the following different advice strategies were generated:

(a) For low-importance expert $e_{1}$ :

$$
\begin{aligned}
& R_{\text {Low }}^{1}=\left\{(i, j) \mid \operatorname{cd}_{i j}<\alpha_{1}\right\}=\left\{(i, j) \mid \operatorname{cd}_{i j}<0.64\right\} \\
& =\{(1,2),(2,1),(2,3),(2,4),(3,1),(3,2),(4,1),(4,2)\} .
\end{aligned}
$$

(b) For medium-importance expert $e_{2}$ : a vector $\boldsymbol{\beta}$ was determined as follows:

$$
\beta_{1}=0.76, \beta_{2}=0.69, \beta_{3}=0.81, \beta_{4}=0.80 .
$$

According to the suggested rules, the preferences that should be modified were identified as

$$
\begin{aligned}
R_{\text {Med }}^{2} & =\left\{(i, j) \mid \mathrm{cd}_{i j}<\alpha_{1} \cap \mathrm{CD}_{i}<\mathrm{CD} \cap \mathrm{PM}_{i}^{k}<\beta_{i}\right\} \\
& =\left\{(i, j) \mid \mathrm{cd}_{i j}<0.64 \cap \mathrm{CD}_{i}<0.645 \cap \mathrm{PM}_{i}^{2}<\beta_{i}\right\} \\
& =\{(1,2),(2,1),(2,3),(2,4)\} .
\end{aligned}
$$

(c) For high-importance experts $e_{3}$ and $e_{4}$ :

$$
\begin{aligned}
R_{\text {High }}^{3}= & \left\{(i, j) \mid \mathrm{cd}_{i j}<\alpha_{1} \cap \mathrm{CD}_{i}<\mathrm{CD}\right. \\
& \left.\cap \mathrm{PM}_{i}^{k}<\beta_{i} \cap \mathrm{pm}_{i j}^{k}<\alpha_{2}\right\} \\
= & \left\{(i, j) \mid \mathrm{cd}_{i j}<0.64 \cap \mathrm{CD}_{i}<0.645\right. \\
& \left.\cap \mathrm{PM}_{i}^{3}<\beta_{i} \cap \mathrm{pm}_{i j}^{3}<0.84\right\}=\varnothing, \\
R_{\text {High }}^{4}= & \varnothing .
\end{aligned}
$$

Finally, the following four different recommendations were suggested to the experts to reach a higher consensus level in the next round:

$$
\begin{aligned}
& \boldsymbol{R}^{1}=\left[\begin{array}{c}
-== \\
+\quad-- \\
++= \\
++=
\end{array}\right], \boldsymbol{R}^{2}=\left[\begin{array}{r}
+== \\
-\quad- \\
== \\
==
\end{array}\right], \\
& \boldsymbol{R}^{3}=\left[\begin{array}{l}
=== \\
=== \\
== \\
==
\end{array}\right], \boldsymbol{R}^{4}=\left[\begin{array}{l}
=== \\
=== \\
== \\
==
\end{array}\right],
\end{aligned}
$$

where $r_{i j}=+,-$, or $=$ denotes that the expert should increase, decrease, or maintain his/her preference accordingly.

Note that the weight for each expert can be altered in each consensus round. We assume that the experts followed the advice and changed their preferences accordingly. In the next round, the whole group reached a higher consensus level of $\mathrm{CD}=0.77$, which was greater than the minimum threshold. The experts' new preference relations were now as follows:

$$
\boldsymbol{E}^{1}=\left[\begin{array}{cccc}
- & \mathrm{MH} & \mathrm{VH} & \mathrm{VH} \\
\mathrm{ML} & - & \mathrm{M} & \mathrm{M} \\
\mathrm{M} & \mathrm{MH} & - & \mathrm{M} \\
\mathrm{M} & \mathrm{M} & \mathrm{M} & -
\end{array}\right],
$$




$$
\begin{aligned}
\boldsymbol{E}^{2} & =\left[\begin{array}{cccc}
- & \text { ML } & \text { ML } & \text { ML } \\
\text { MH } & - & \text { M } & \text { MH } \\
\text { MH } & \text { L } & - & \text { M } \\
\text { H } & \text { VL } & \text { M } & -
\end{array}\right], \\
\boldsymbol{E}^{3} & =\left[\begin{array}{cccc}
- & \text { M } & \text { ML } & \text { ML } \\
\text { M } & - & \text { L } & \text { L } \\
\text { H } & \text { H } & - & \text { M } \\
\text { MH } & \text { MH } & \text { M } & -
\end{array}\right], \\
\boldsymbol{E}^{4} & =\left[\begin{array}{cccc}
- & \text { M } & \text { ML } & \text { ML } \\
\text { M } & - & \text { L } & \text { L } \\
\text { H } & \text { H } & - & \text { M } \\
\text { MH } & \text { MH } & \text { M } & -
\end{array}\right] .
\end{aligned}
$$

The new aggregated group preference matrix was the following:

$$
\tilde{\tilde{\boldsymbol{P}}}_{\text {new }}=\left[\begin{array}{cccc}
- & \tilde{\tilde{p}}_{12} & \tilde{\tilde{p}}_{13} & \tilde{\tilde{p}}_{14} \\
\tilde{\tilde{p}}_{21} & - & \tilde{\tilde{p}}_{23} & \tilde{\tilde{p}}_{24} \\
\tilde{\tilde{p}}_{31} & \tilde{\tilde{p}}_{32} & - & \tilde{\tilde{p}}_{34} \\
\tilde{\tilde{p}}_{41} & \tilde{\tilde{p}}_{42} & \tilde{\tilde{p}}_{43} & -
\end{array}\right],
$$

where

$$
\begin{aligned}
\tilde{\tilde{p}}_{12}= & ((0.30,0.50,0.50,0.70 ; 1,1), \\
& (0.40,0.50,0.50,0.60 ; 0.9,0.9)), \\
\tilde{\tilde{p}}_{13}= & ((0.29,0.47,0.47,0.62 ; 1,1), \\
& (0.38,0.47,0.47,0.54 ; 0.9,0.9)), \\
\tilde{\tilde{p}}_{14}= & ((0.29,0.47,0.47,0.62 ; 1,1), \\
& (0.38,0.47,0.47,0.54 ; 0.9,0.9)), \\
\tilde{\tilde{p}}_{21}= & ((0.30,0.50,0.50,0.70 ; 1,1), \\
& (0.40,0.50,0.50,0.60 ; 0.9,0.9)), \\
\tilde{\tilde{p}}_{23}= & ((0.14,0.29,0.29,0.49 ; 1,1), \\
& (0.22,0.29,0.29,0.39 ; 0.9,0.9)), \\
\tilde{\tilde{p}}_{24}= & ((0.19,0.34,0.34,0.54 ; 1,1), \\
& (0.26,0.34,0.34,0.44 ; 0.9,0.9)), \\
\tilde{\tilde{p}}_{31}= & ((0.56,0.76,0.76,0.90 ; 1,1), \\
& (0.66,0.76,0.76,0.83 ; 0.9,0.9)), \\
\tilde{\tilde{p}}_{32}= & ((0.49,0.66,0.66,0.81 ; 1,1), \\
& (0.58,0.66,0.66,0.74 ; 0.9,0.9)),
\end{aligned}
$$

$$
\begin{aligned}
\tilde{\tilde{p}}_{34}= & ((0.3,0.5,0.5,0.70 ; 1,1), \\
& (0.4,0.5,0.5,0.6 ; 0.9,0.9)), \\
\tilde{\tilde{p}}_{41}= & ((0.50,0.70,0.70,0.88 ; 1,1), \\
& (0.60,0.70,0.70,0.79 ; 0.9,0.9)), \\
\tilde{\tilde{p}}_{42}= & ((0.33,0.49,0.49,0.66 ; 1,1), \\
& (0.41,0.49,0.49,0.58 ; 0.9,0.9)), \\
\tilde{\tilde{p}}_{43}= & ((0.3,0.5,0.5,0.70 ; 1,1), \\
& (0.4,0.5,0.5,0.6 ; 0.9,0.9)) .
\end{aligned}
$$

\section{Selection process}

Finally, the selection process was applied to select the appropriate solution using the dominance degree based on the rank value of each alternative, using Eq. (27). Thus, we obtained

$$
x_{1}=19.51, x_{2}=17.76, x_{3}=22.37, x_{4}=21.02 \text {. }
$$

As such, the final ranking list was $x_{2}<x_{1}<x_{4}<x_{3}$, and therefore $x_{3}$ was chosen to be the recommended solution.

\subsection{Other consensus models}

\subsubsection{Statistical study}

As mentioned in Section 3.1, different similarity matrices must be calculated to check the consensus measures, wherein different distance functions are used. Meanwhile, various distance functions have been used in existing studies. Therefore, it is worth carrying out some studies to determine whether different distance functions are able to influence the consensus-reaching process. To this aim, we apply five different distance functions commonly used in existing studies. These are the Manhattan, Euclidean, Cosine, Dice, and Jaccard distance functions (Chiclana et al., 2013).

Using the same preference values as in the above example, we conducted our experiment using the above five distance functions, and five different consensus measures at the relation level were obtained (Table 2).

It can be seen that the Cosine function produced the largest consensus level, followed by the Dice, Manhattan, Euclidean, and Jaccard distance functions. Moreover, the Manhattan and the Euclidean distance functions produced similar results. Thus, decision makers can use a specific distance function to reach a 
Table 2 Consensus measures at the relation level using different distance functions

\begin{tabular}{cc||cc}
\hline Distance function & CD & Distance function & CD \\
\hline Manhattan & 0.6458 & Dice & 0.6736 \\
Euclidean & 0.6408 & Jaccard & 0.6004 \\
Cosine & 0.9108 & & \\
\hline
\end{tabular}

CD: consensus measure on the relation

higher consensus level based on these comparative results, which can lead to a faster convergence.

Chiclana et al. (2013) carried out similar comparative statistical studies under different combinations of numbers of experts and linguistic ordered weighted averaging (OWA) operators. Their results were similar.

\subsubsection{Comparative study}

To demonstrate clearly the feasibility and practicality of our proposed model, three other consensus models were applied under the same assumptions. These were a Web-based consensus support system (Alonso et al., 2010), a linguistic consensus model (Alonso et al., 2013), and a trust-based consensus model (Wu et al., 2015).

The first model develops a Web-based consensus support system, based on consistency and consensus measures, and we found that it was good at maintaining individual consistency. The second model incorporates some delegation and a feedback mechanism to quicken the consensus-reaching process, and it was designed particularly to work in highly dynamic environments. The third model uses a trustbased estimation and aggregation method within a network social group.

Some reasonable hypotheses have been assumed while conducting the experiments. For example, the parameter to balance the weight of consensus and consistency criteria in the first model was set to 0.5 , while the consistency level was set to 0.7 . The trust weights in the second model and the average trust degrees for each expert were all settled as 0.25 . After careful computations, the following three ranking results were obtained:
(a) $x_{4}<x_{1}<x_{3}<x_{2}$,
(b) $x_{1}<x_{4}<x_{2}<x_{3}$,
(c) $x_{4}<x_{1}<x_{2}<x_{3}$

The first model yielded a very different result from our model. The second alternative, $x_{2}$, was found to be the best choice, while $x_{3}$ came only in the second place. However, according to the opinions of the high-level importance experts in our proposed model, $e_{3}$ and $e_{4}, x_{3}$ should be superior to $x_{2}$. For the second and third models, the best alternative remained the same as in our model, but the ranking of the others varied significantly. The differences may be due to the different methods used to compute the weights. Moreover, variations in the determination of the corresponding parameters in the different models can affect the results. However, none of the three consensus models considered the experts' weights when generating advice to the experts, and a greater number of consensus rounds were necessary to achieve a satisfactory consensus level. Thus, it is a logical and necessary procedure to distinguish the experts throughout the consensus-reaching process.

\section{Conclusions}

A new consensus model for GDM problems, using an IT2 fuzzy environment, is proposed. In our model, experts are asked to express their preferences using linguistic terms, which are characterized by IT2 FSs. Different weights for the experts are determined based on the experts' opinions on the problem. Note that the weights do not necessarily remain the same in each consensus round. Two criteria are used simultaneously to guide the consensus-reaching process, and we propose a new feedback mechanism that generates different advice strategies based on the levels of the experts' knowledge concerning the specific problem (Zhang et al., 2015). In general, experts with a lower level of importance receive more advice, and experts with a higher level of importance should alter their opinions less. To the best of our knowledge, this is the first time that a feedback mechanism that considers differences in experts' knowledge levels in an IT2 fuzzy environment has been applied to a consensus model, which can certainly provide us with greater insight into the GDM process.

The following points summarize the advantages of our consensus model compared with other existing models:

1. The IT2 FSs used in this study can depict information with more vagueness and uncertainty, 
which can provide decision makers with more flexibility, and they can be used in other decision-making fields.

2. The automatic feedback mechanism, which is designed to generate specific advice to aid the consensus-reaching process, overcomes the problem of a traditional moderator. In addition, it yields promising results in practice.

3. The concept of the importance of the experts is considered throughout the decision-making process, which is one of the main novelties in this study. Furthermore, we believe that this is a reasonable addition to the model, and it can lead to a faster convergence.

However, the following disadvantages are also worth noting:

1. The consensus model cannot guarantee convergence. The feedback mechanism is responsible only for generating some suggestions to the experts to reach a higher consensus degree. However, it is the experts' decision whether to accept it or not. In the future, some principles of persuasion and weapons of influence could be applied, to support the consensusreaching process (Herrera-Viedma et al., 2014).

2. This consensus model fails to detect or forecast the consistency of the experts, which may lead to unreasonable results. Therefore, we intend to apply some mechanisms that can effectively check the consistency of the experts in a future study (Pérez et al., 2014).

3. One of the main drawbacks of our method lies in its computational difficulty. In this regard, creating some new consensus measures, along with the use of different distance functions, would be interesting.

In summary, there are still some new challenges associated with GDM problems that need to be solved. In particular, we should focus more on some new consensus approaches, particularly in social networks, due to their new characteristics and features in realworld applications (Cabrerizo et al., 2015b). Some initial efforts have been attempted in this direction (Wu and Chiclana, 2014; Wu et al., 2015). New preference structures applied in consensus approaches to represent the individuals' preferences should be given enough attention. Some visualization tools and the development of software systems could be combined in the consensus-reaching process, to support a better understanding of different states and help reach a high consensus level (Cabrerizo et al., 2015b). We believe that these new challenges will help make this topic a hot one in the future.

\section{References}

Alonso, S., Herrera-Viedma, E., Chiclana, F., et al., 2010. A web based consensus support system for group decision making problems and incomplete preferences. Inform. Sci., 180(23):4477-4495. http://dx.doi.org/10.1016/j.ins.2010.08.005

Alonso, S., Pérez, I.J., Cabrerizo, F.J., et al., 2013. A linguistic consensus model for Web 2.0 communities. Appl. Soft Comput., 13(1):149-157. http://dx.doi.org/10.1016/j.asoc.2012.08.009

Cabrerizo, F.J., Moreno, J.M., Pérez, I.J., et al., 2010. Analyzing consensus approaches in fuzzy group decision making: advantages and drawbacks. Soft Comput., 14(5):451-463.

http://dx.doi.org/10.1007/s00500-009-0453-x

Cabrerizo, F.J., Herrera-Viedma, E., Pedrycz, W., 2013. A method based on PSO and granular computing of linguistic information to solve group decision making problems defined in heterogeneous contexts. Eur. J. Oper. Res., 230(3):624-633. http://dx.doi.org/10.1016/j.ejor.2013.04.046

Cabrerizo, F.J., Morente-Molinera, J.A., Pérez, I.J., et al., 2015a. A decision support system to develop a quality management in academic digital libraries. Inform. Sci., 323:48-58. http://dx.doi.org/10.1016/j.ins.2015.06.022

Cabrerizo, F.J., Chiclana, F., Al-Hmouz, R., et al., 2015 b. Fuzzy decision making and consensus: challenges. J. Intell. Fuzzy Syst., 29(3):1109-1118. http://dx.doi.org/10.3233/IFS-151719

Chen, S.M., Lee, L.W., 2010. Fuzzy multiple attributes group decision-making based on the ranking values and the arithmetic operations of interval type-2 fuzzy sets. Expert Syst. Appl., 37(1):824-833. http://dx.doi.org/10.1016/j.eswa.2009.06.094

Chen, Z., Yang, W., 2011. A new multiple attribute group decision making method in intuitionistic fuzzy setting. Appl. Math. Model., 35(9):4424-4437. http://dx.doi.org/10.1016/j.apm.2011.03.015

Chiclana, F., Tapia Garcia, J.M., Del Moral, M.J., et al., 2013. A statistical comparative study of different similarity measures of consensus in group decision making. Inform. Sci., 221:110-123.

http://dx.doi.org/10.1016/j.ins.2012.09.014

Feng, Z.Q., Liu, C., Huang, H., 2014. Knowledge modeling based on interval-valued fuzzy rough set and similarity inference: prediction of welding distortion. J. Zhejiang Univ.-Sci. C (Comput. \& Electron.), 15(8):636-650. http://dx.doi.org/10.1631/jzus.C1300370

Herrera-Viedma, E., Alonso, S., Chiclana, F., et al., 2007. A consensus model for group decision making with incomplete fuzzy preference relations. IEEE Trans. Fuzzy Syst., 15(5):863-877. http://dx.doi.org/10.1109/TFUZZ.2006.889952 
Herrera-Viedma, E., Cabrerizo, F.J., Kacprzyk, J., et al., 2014. A review of soft consensus models in a fuzzy environment. Inform. Fus., 17:4-13. http://dx.doi.org/10.1016/j.inffus.2013.04.002

Lee, L.W., Chen, S.M., 2008. A new method for fuzzy multiple attributes group decision-making based on the arithmetic operations of interval type-2 fuzzy sets. Proc. 7 th Int. Conf. on Machine Learning and Cybernetics, p.3084-3089. http://dx.doi.org/10.1109/ICMLC.2008.4620938

Mata, F., Martínez, L., Herrera-Viedma, E., 2009. An adaptive consensus support model for group decision-making problems in a multigranular fuzzy linguistic context. IEEE Trans. Fuzzy Syst., 17(2):279-290. http://dx.doi.org/10.1109/TFUZZ.2009.2013457

Mendel, J.M., John, R.I., Liu, F., 2006. Interval type-2 fuzzy logic systems made simple. IEEE Trans. Fuzzy Syst., 14(6):808-821. http://dx.doi.org/10.1109/TFUZZ.2006.879986

Mendel, J.M., Zadeh, L.A., Trillas, E., et al., 2010. What computing with words means to me. IEEE Comput. Intell. Mag., 5(1):20-26. http://dx.doi.org/10.1109/MCI.2009.934561

Moharrer, M., Tahayori, H., Livi, L., et al., 2015. Interval type-2 fuzzy sets to model linguistic label perception in online services satisfaction. Soft Comput., 19(1):237-250. http://dx.doi.org/10.1007/s00500-014-1246-4

Pérez, I.J., Cabrerizo, F.J., Alonso, S., et al., 2014. A new consensus model for group decision making problems with non-homogeneous experts. IEEE Trans. Syst. Man Cybern. Syst., 44(4):494-498. http://dx.doi.org/10.1109/TSMC.2013.2259155

Sabahi, F., Akbarzadeh-T, M.R., 2014. A framework for analysis of extended fuzzy logic. J. Zhejiang Univ.-Sci. C
(Comput. \& Electron.), 15(7):584-591. http://dx.doi.org/10.1631/jzus.C1300217

Wang, W., Liu, X., Qin, Y., 2012. Multi-attribute group decision making models under interval type-2 fuzzy environment. Knowl.-Based Syst., 30:121-128. http://dx.doi.org/10.1016/j.knosys.2012.01.005

Wang, Z.J., Li, K.W., 2015. A multi-step goal programming approach for group decision making with incomplete interval additive reciprocal comparison matrices. Eur. J. Oper. Res., 242(3):890-900. http://dx.doi.org/10.1016/j.ejor.2014.10.025

$\mathrm{Wu}, \mathrm{D} .$, Mendel, J.M., 2011. On the continuity of type-1 and interval type-2 fuzzy logic systems. IEEE Trans. Fuzzy Syst., 19(1):179-192. http://dx.doi.org/10.1109/TFUZZ.2010.2091962

Wu, J., Chiclana, F., 2014. A social network analysis trust-consensus based approach to group decision-making problems with interval-valued fuzzy reciprocal preference relations. Knowl.-Based Syst., 59:97-107. http://dx.doi.org/10.1016/j.knosys.2014.01.017

Wu, J., Chiclana, F., Herrera-Viedma, E., 2015. Trust based consensus model for social network in an incomplete linguistic information context. Appl. Soft Comput., 35:827-839. http://dx.doi.org/10.1016/j.asoc.2015.02.023

Zhang, X., Ge, B., Jiang, J., et al., 2015. A new consensus model for group decision making using fuzzy linguistic preference relations with heterogeneous experts. J. Intell. Fuzzy Syst., 30(1):171-182. http://dx.doi.org/10.3233/IFS-151744

Zhang, Z., Zhang, S., 2013. A novel approach to multi attribute group decision making based on trapezoidal interval type-2 fuzzy soft sets. Appl. Math. Model., 37(7): 4948-4971.

http://dx.doi.org/10.1016/j.apm.2012.10.006 